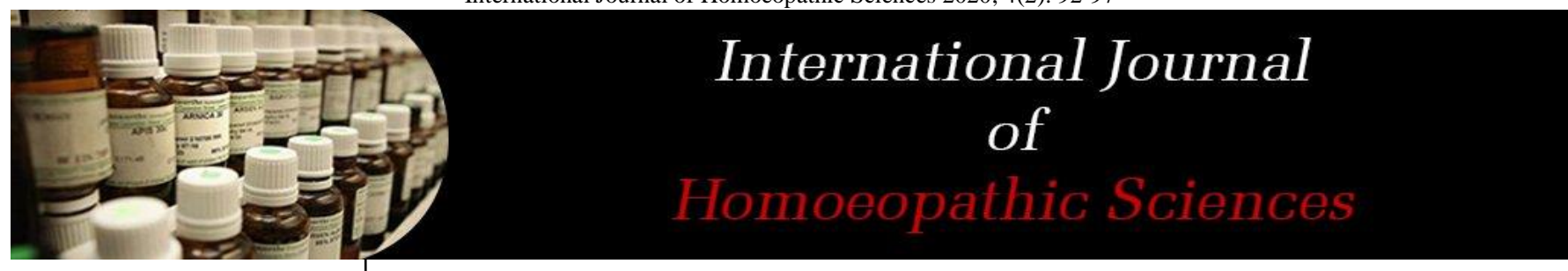

E-ISSN: 2616-4493 P-ISSN: 2616-4485 www.homoeopathicjournal.com IJHS 2020; 4(2): 92-97 Received: 07-02-2020 Accepted: 09-03-2020

Gurudev Choubey Research Officer (H), Central Council for Research in Homoeopathy, Ministry of AYUSH, Govt. of India

Dhiraj Debnath Senior Research, Central Council for Research in Homoeopathy, Ministry of AYUSH, Govt. of India

Varanasi Roja Research Officer (H), Central Council for Research in Homoeopathy, Ministry of AYUSH, Govt. of India

Jaya Gupta

Research Officer (H), Central

Council for Research in Homoeopathy, Ministry of AYUSH, Govt. of India

Abhiram Banerjee Research Associate (H), Central Council for Research in Homoeopathy, Ministry of AYUSH, Govt. of India
Corresponding Author: Gurudev Choubey Research Officer(H), Central Council for Research in Homoeopathy, Ministry of AYUSH, Govt. of India

\section{Managing pain and stiffness through individualized homoeopathy in lumbar spondylosis: Results of a prospective consecutive case series}

\author{
Gurudev Choubey, Dhiraj Debnath, Varanasi Roja, Jaya Gupta and \\ Abhiram Banerjee
}

DOI: https://doi.org/10.33545/26164485.2020.v4.i2b.153

\begin{abstract}
Background: Low back pain affects approximately $60-85 \%$ of adults during some point in their lives and LS is responsible for about $10 \%$ of all the back-pain. Common conventional treatments like NSAIDS and Epidural steroid injections (ESI) deleterious side effects.

Objectives: This case series of lumbar spondylosis presented here tries to show response to individualized homoeopathic medicine in context of pain, stiffness through Visual Analog Scale (VAS) and RODQ.

Method: Patients with spondylotic changes in lumbar spine were included in this case series. Individualized homoeopathic medicines were prescribed in singledose in each case and followed up every 2 weeks for eight months. Pain and stiffness in every case was assessed using Visual Analog Scale (VAS) at baseline and every two weeks for eight months. Revised Oswestry disability questionnaire (RODQ) scoresto assess quality of life was used at baseline and at the end of 8 months. Results: Mean VAS score for pain reduced from 78.07 $\pm 13.72 \mathrm{~mm}$ (baseline)to $38 \pm 25.55 \mathrm{~mm}$ (end); 95\% CI: 22.05 - 58.09; $P<0.05$. Stiffness reduced from $65.10 \pm 16.35$ (baseline) to $16.50 \pm 10.01 \mathrm{~mm}$ (end); 95\% CI: $34.88-62.32 ; P<0.05$. Mean RODQ score reduced from $31.64 \pm 3.20$ (baseline) to $13.57 \pm 7.06$ (end); $95 \%$ CI: $13.21-22.93 ; P<0.05$.
\end{abstract}

Conclusion: The results has trend showing positive role of homoeopathic medicines in management symptoms related to lumbar spondylosis. Controlled clinical studies are warranted.

Keywords: Case series, lumbar spondylosis, homoeopathy, RODQ

\section{Introduction}

Lumbar Spondylosis (LS) is defined as degenerative condition affecting the discs, vertebral bodies and associated joints of the lumbar spine. Low back pain affects approximately 60 $85 \%$ of adults during some point in their lives and LS is responsible for about $10 \%$ of all the backpain conditions ${ }^{[1,2]}$. Conventional medical treatment like NSAIDS and Epidural steroid injections (ESI) have become a common interventional strategy in the management of lumbar spondylosis and these medications are having deleterious effects like large intestinal ulcers, bleeding, perforation, non-specific colitis and meningitis, arachnoiditis respectively. ${ }^{3-}$ ${ }^{4} \mathrm{CAM}$ (Complementary/ Alternative medicine) therapy is being popular in patients with musculoskeletal disorders including low back pain ${ }^{[5,6]}$ A high prevalence of CAM use in rheumatic patients is observed in the United States(18-94\%), Canada (60-91\%), Mexico(56-83\%), Australia (40-82\%), Germany (78\%) and India (43-72\%) ${ }^{[7]}$.

There are very few papers published for effectiveness of homoeopathy in low back pain (LBP) ${ }^{[8,9]}$ but there is paucity of studies specifically regarding the homoeopathic treatment of LS ${ }^{[10]}$. In one study it has been shown that homoeopathy is efficacious in significant decrease of Oswestry score of LBP in compared to standardized physiotherapy and in another study, it was seen that classical homeopathic treatment represented an effective treatment for low back pain and it improved health-related QoL and reduces the use of other healthcare services. This paper presents a series of 17 consecutive cases clinically and radio logically diagnosed as lumbar spondylosis and their response to individualized homoeopathic medicine in context of pain, stiffness functional disability through Visual Analog Scale (VAS) ${ }^{[11]}$ and Revised Oswestry disability questionnaire (RODQ) ${ }^{[12-15]}$ for quality of life. 


\section{Methodology \\ Study design}

Consecutive cases from Rheumatology Out Patient Department (OPD) at Clinical Research Unit (H), Siliguri were examined through detailed case taking including clinical examination of each and every patient in a prestructured proforma. In this prospective case series, patients aged more than 30 years attending the OPD with spondylotic changes on radiological evidence (X-ray) of lumbar spine were considered.

\section{Outcome measures}

VAS (Visual Analog Scale) ${ }^{[9]}$ in a scale of 0 to $100 \mathrm{~mm}$ ('0'score indicate no symptom whereas $100 \mathrm{~mm}$ indicates the worst possible symptom) for pain, stiffness was assessed before treatment (baseline) and till 8 months. Revised Oswestry Low Back Pain Disability Questionnaire (RODQ) were assessed was for disability and quality of life which enables to understand how much your low back pain has affected your ability to manage your everyday activities, was assessed be for treatment and end of the treatment ${ }^{[12-14]}$, In this questionnaire there are 10 sections ( 0 to 5 points each) for pain intensity, Personal Care, Lifting, Walking, Sitting, Standing, Sleeping, Social life, Travel and Changing of Pain. Each of the 10 sections is scored separately (0 to 5 points each) and then added up ( $\max$ total $=50)$. The percentage of disability was calculated as: Patient's Score / total possible scores $\mathrm{x} 100=$ $\%$ Disability

\section{Intervention}

A detailed case taking was done in every case and an individualised homoeopathic medicine was selected according to homoeopathic principles. The potency was used as per the need of the patients in every case and the doses were selected as per the case requirements. The repetition of medicine was done as per instruction stated in Organon of Medicine.

\section{Statistical analysis}

Paired " $t$ " test was used to analyse the changes that occurred in the values of "VAS scoring system" and Revised Oswestry low back pain disability questionnaire scores as a result of the homoeopathic intervention. Statistical package for the social sciences (SPSS) software is used for statistical calculations.

\section{Results}

The descriptive data of all the 17 patients have been shown in the table no. 1. VAS scores of pains, stiffness of every month have been mentioned in table no $2 \& 3$ and the course of pain and stiffness (mean values) of LS over 8 months has been shown in a line diagram in fig. no.1.RODQ scores have been mentioned in table no. 4 \&5 and the interpretation of disability score has been shown in fig. 2 . The results of statistical analysis have been displayed in table no. 6.

Table 1: Summary of presentations of patients with Lumbar spondylosis

\begin{tabular}{|c|c|c|c|c|c|c|c|}
\hline $\begin{array}{l}\text { Case } \\
\text { No. }\end{array}$ & Age & Gender & Occupation & Initial presentations & $\begin{array}{c}\text { VAS } \\
\text { baseline }\end{array}$ & $\begin{array}{l}\text { Associated } \\
\text { Medical } \\
\text { Conditions }\end{array}$ & $\begin{array}{c}\text { Prescribed Homoeopathic } \\
\text { Medicines }\end{array}$ \\
\hline 1 & $\begin{array}{c}53 \\
\text { years }\end{array} \mid$ & Female & House wife & $\begin{array}{l}\text { Pain in the lower back, }<\text { from } \\
\text { standing, evening with H/O trauma } \\
\text { before } 5-6 \text { months, father- MI, }\end{array}$ & $\begin{array}{c}\text { Pain- } 49 \\
\text { mm } \\
\text { Stiffness- } 0\end{array}$ & \begin{tabular}{|c|} 
Dyspepsia \\
Hypertension, \\
Grade I \\
Spondylolysthesis, \\
Calcaneal spur \\
\end{tabular} & $\begin{array}{l}\text { Nuxvom 200C (as an acute for } \\
\text { farting problems) } \\
\text { Arnica mont } 1 \mathrm{M} \text { (for remaining of } \\
\text { symptoms including h/o trauma) }\end{array}$ \\
\hline 2 & $\begin{array}{c}45 \\
\text { years }\end{array}$ & Female & House keeper & $\begin{array}{l}\text { Pain in back, knees and both shoulder } \\
\text { joints, < at night, morning, H/O } \\
\text { suppressed skin eruption, Father-BHP }\end{array}$ & \begin{tabular}{|c|} 
Pain- 60 \\
mm \\
Stiffness- 0
\end{tabular} & $\begin{array}{l}\text { Dyspepsia } \\
\text { OA knee }\end{array}$ & $\begin{array}{c}\text { Sulphur 200C } \\
\text { Pulsatilla 30C(as an acute for } \\
\text { farting problems) }\end{array}$ \\
\hline 3 & $\begin{array}{c}69 \\
\text { years }\end{array}$ & Female & House wife & $\begin{array}{c}\text { Pain in lumbar spine, in both knees < } \\
\text { walking, standing,> by motion H/O- } \\
\text { hysterectomy, }\end{array}$ & $\begin{array}{c}\text { Pain-70 mm } \\
\text { Stiffness-0 }\end{array}$ & $\begin{array}{l}\text { Deafness, } \\
\text { Dyspepsia, } \\
\text { Wrist Ganglion }\end{array}$ & $\begin{array}{c}\text { Rhus tox 200C, 1M. } \\
\text { Calc fluor 200C(improvement was } \\
\text { stopped and after recasetaking } \\
\text { pain was still }>\text { by motion, warm } \\
\text { application } \&<\text { byrest and } \\
\text { presence of wrist ganglion) }\end{array}$ \\
\hline 4 & $\begin{array}{c}63 \\
\text { years }\end{array} \mid$ & Female & House wife & $\begin{array}{c}\text { Pain in lower back and in both knees < } \\
\text { bending forward, > by pressure, } \\
\text { warmth, H/O- hysterectomy }\end{array}$ & $\begin{array}{c}\text { Pain-70 mm } \\
\text { Stiffness-0 }\end{array}$ & $\begin{array}{c}\text { DM, Hypertension, } \\
\text { Hypothyroidism }\end{array}$ & Sulphur 6C, 30C, 200C \\
\hline 5 & $\begin{array}{c}37 \\
\text { years }\end{array}$ & Male & $\begin{array}{l}\text { Data entry } \\
\text { operator }\end{array}$ & $\begin{array}{c}\text { Pain in lumbo-sacral region < by rest, } \\
\text { morning }>\text { by pressure and in evening, } \\
\text { H/O jaundice, father- Hypertension, } \\
\text { DM }\end{array}$ & $\begin{array}{c}\text { Pain- } 60 \mathrm{~mm} \\
\text { Stiffness-40 } \\
\mathrm{mm}\end{array}$ & $\begin{array}{l}\text { Lumbar scoliosis, } \\
\text { Bilateral sacroilitis }\end{array}$ & Sulphur 6C, 30C, 200C \\
\hline 6 & $\begin{array}{c}80 \\
\text { years }\end{array}$ & Male & $\begin{array}{c}\text { Retired } \\
\text { service man }\end{array}$ & $\begin{array}{c}\text { Stitching pain in lower back and } \\
\text { weakness of lower limb < pressure, } \\
\text { H/O- typhoid, malaria; father- asthma }\end{array}$ & $\begin{array}{c}\text { Pain- } 80 \mathrm{~mm} \\
\text { Stiffness-0 }\end{array}$ & Lumbar scoliosis & Pulsatilla 6C, 30C \\
\hline 7 & $\begin{array}{c}52 \\
\text { years }\end{array} \mid$ & Female & House wife & $\begin{array}{c}\text { Pain in lumbar spine < rest, rising } \\
\text { from sitting, > by motion, cold; H/O- } \\
\text { Cholecystectomy, Father- DM, } \\
\text { hypertension }\end{array}$ & $\begin{array}{c}\text { Pain-70 mm } \\
\text { Stiffness-0 }\end{array}$ & Insomnia & Pulsatilla 6C \\
\hline 8 & $\begin{array}{c}42 \\
\text { years }\end{array} \mid$ & Female & House wife & $\begin{array}{l}\text { Pain in back and in both knees < new } \\
\text { and full moon, first motion, H/O- } \\
\text { suppressed skin eruption, mother- } \\
\text { asthma }\end{array}$ & $\begin{array}{c}\text { Pain- } 80 \mathrm{~mm} \\
\text { Stiffness-70 } \\
\mathrm{mm}\end{array}$ & $\begin{array}{c}\text { OA Knee } \\
\text { Dyspepsia, } \\
\text { Bilateral sacroilitis }\end{array}$ & Lycopodium 6C \\
\hline 9 & \begin{tabular}{c|}
57 \\
years
\end{tabular} & Male & Driver & $\begin{array}{l}\text { Pain in lower back and nape of neck < } \\
\text { rest, morning, > by continued motion; }\end{array}$ & \begin{tabular}{|l|} 
Pain- $88 \mathrm{~mm}$ \\
Stiffness-40
\end{tabular} & $\begin{array}{c}\text { Diffuse idiopathic } \\
\text { skeletal }\end{array}$ & Rhus tox $30 \mathrm{C}$ \\
\hline
\end{tabular}




\begin{tabular}{|c|c|c|c|c|c|c|c|}
\hline & & & & F/H: mother- TB & $\mathrm{mm}$ & $\begin{array}{l}\text { hyperostosis } \\
\text { (DISH) }\end{array}$ & \\
\hline 10 & $\begin{array}{c}50 \\
\text { years }\end{array}$ & Male & Painter & $\begin{array}{c}\text { Pain in back and in both knees < rising } \\
\text { from sitting,> by warmth, motion; } \\
\text { H/O- trauma to back, mother- MI }\end{array}$ & $\begin{array}{c}\text { Pain- } 83 \mathrm{~mm} \\
\text { Stiffness-70 } \\
\mathrm{mm}\end{array}$ & OA knee & $\begin{array}{c}\text { Arnica 200C( for h/o trauma) } \\
\text { Rhus tox 6C, 30C, } \\
\text { 200C(improvement was stopped } \\
\text { \& depending on remaing } \\
\text { symptoms) }\end{array}$ \\
\hline 11 & $\begin{array}{c}39 \\
\text { years }\end{array}$ & Male & Businessman & $\begin{array}{c}\text { Pain lower back and left ankle < cold } \\
\text { weather, exertion, H/O- cataract } \\
\text { surgery, father- DM }\end{array}$ & \begin{tabular}{|c|}
$\begin{array}{c}\text { Pain- } 88 \mathrm{~mm} \\
\text { Stiffness-60 } \\
\mathrm{mm}\end{array}$ \\
\end{tabular} & Absent & $\begin{array}{c}\text { Phosphorus 30C } \\
\text { Rhus tox 30C(for acute } \\
\text { exacerbation of pain) }\end{array}$ \\
\hline 12 & $\begin{array}{c}65 \\
\text { years }\end{array}$ & Male & $\begin{array}{c}\text { Retired } \\
\text { service man }\end{array}$ & $\begin{array}{c}\text { Pain in back } \\
<\text { motion, cold ,> by rest, warmth } \\
\text { H/O- CVA, mother- cancer }\end{array}$ & \begin{tabular}{|c|} 
Pain-94 mm \\
Stiffness-90 \\
$\mathrm{mm}$
\end{tabular} & Lumbar scoliosis & $\begin{array}{c}\text { Bryonia 30C(as an acute remedy } \\
\text { for pain) } \\
\text { Calcarea carb 200C } \\
\end{array}$ \\
\hline 13 & $\mid \begin{array}{c}59 \\
\text { years }\end{array}$ & Female & House wife & $\begin{array}{c}\text { Pain in back and both knees < night, } \\
\text { motion, > by lying down, warmth; } \\
\text { H/O-malaria, parents- DM }\end{array}$ & $\left|\begin{array}{c}\text { Pain- } 87 \mathrm{~mm} \\
\text { Stiffness-82 } \\
\mathrm{mm}\end{array}\right|$ & $\begin{array}{c}\text { OA knee } \\
\text { Dyspepsia }\end{array}$ & \begin{tabular}{|c|} 
Lycopodium 30C, 200C. \\
Arnica 200, 1M(not improving \\
further \& after recasetaking h/o of \\
injury was found)
\end{tabular} \\
\hline 14 & $\begin{array}{c}47 \\
\text { years }\end{array}$ & Male & Farmer & $\begin{array}{l}\text { Pain in lower back < evening, } \\
\text { exertion, > by rest, warmth, pressure; } \\
\text { F/H: mother- hypertension, allergy }\end{array}$ & $\left|\begin{array}{c}\text { Pain- } 87 \mathrm{~mm} \\
\text { Stiffness-78 } \\
\mathrm{mm}\end{array}\right|$ & Dyspepsia & $\begin{array}{c}\text { Phosphorus 30C, 200C } \\
\text { Rhus tox 30C(as an acute } \\
\text { exacerbation of pain due to h/o } \\
\text { lifting heavy weight) }\end{array}$ \\
\hline 15 & $\begin{array}{c}43 \\
\text { years }\end{array}$ & Female & House wife & $\begin{array}{c}\text { Pain in lower back, in both knees and } \\
\text { left shoulder joint < rising from } \\
\text { sitting, standing, > by motion; H/O- } \\
\text { Cholecystectomy, parents- } \\
\text { hypertension, DM }\end{array}$ & $\left|\begin{array}{c}\text { Pain- } 87 \mathrm{~mm} \\
\text { Stiffness-67 } \\
\mathrm{mm}\end{array}\right|$ & $\begin{array}{l}\text { Tinea Corporis } \\
\text { Dyspepsia } \\
\text { OA knee }\end{array}$ & $\begin{array}{c}\text { Bacillinum 200C } \\
\text { Sulphur 30C, 200C(improvement } \\
\text { was stopped and after recasetaking } \\
\text { it was found pain in left shoulder } \\
\text { and LBP < standing \& > by lying } \\
\text { on right side) }\end{array}$ \\
\hline 16 & $\begin{array}{c}65 \\
\text { years }\end{array} \mid$ & Female & House wife & $\begin{array}{c}\text { Pain in lumbar region, cervical region } \\
\text { and in both knees < at night, rest, > by } \\
\text { motion; H/O- trauma to back }\end{array}$ & $\begin{array}{c}\text { Pain- } 83 \mathrm{~mm} \\
\text { Stiffness- } 62 \\
\mathrm{~mm}\end{array}$ & OA knee & \begin{tabular}{|c|} 
Rhus tox 200 \\
Arnica $1 \mathrm{M}$ (not improving further \\
$\&$ then h/o of injury was included)
\end{tabular} \\
\hline 17 & $\left|\begin{array}{c}50 \\
\text { years }\end{array}\right|$ & Male & Business man & $\begin{array}{c}\text { Pain in lumbar and cervical spine < } \\
\text { rising from sitting, early morning, } \\
\text { night, > by motion; H/O- ring worm, } \\
\text { parents are hypertensive }\end{array}$ & $\begin{array}{c}\text { Pain-87 mm } \\
\text { Stiffness-62 } \\
\mathrm{mm}\end{array} \mid$ & $\begin{array}{c}\text { Cervical } \\
\text { spondylosis }\end{array}$ & Lycopodium 30C, 200C \\
\hline
\end{tabular}

Table 2: showing every month VAS scores ( $\mathrm{mm}$ ) of pain

\begin{tabular}{|c|c|c|c|c|c|c|c|c|c|}
\hline Case no. & Base line & $\mathbf{1}^{\text {st }}$ & $\mathbf{2}^{\text {nd }}$ & $\mathbf{3}^{\text {rd }}$ & $\mathbf{4 t h}$ & $\mathbf{5}^{\text {th }}$ & $\mathbf{6 t h}$ & 7th $^{\text {8th }}$ \\
\hline 1. & 49 & 60 & 55 & 48 & 30 & 24 & 20 & 12 & 7 \\
\hline 2. & 60 & 65 & 70 & 70 & 70 & 74 & 80 & 85 & 89 \\
\hline 3. & 70 & 75 & 71 & 65 & 50 & 45 & 35 & 20 & 10 \\
\hline 4. & -- & -- & -- & -- & -- & -- & -- & -- & --- \\
\hline 5. & 60 & 60 & 60 & 65 & 65 & 60 & 50 & 50 & 50 \\
\hline 6. & -- & -- & -- & -- & -- & -- & -- & -- & --- \\
\hline 7. & 70 & 70 & 70 & 70 & 70 & 65 & 65 & 65 & 65 \\
\hline 8. & -- & -- & -- & -- & -- & -- & -- & -- & -- \\
\hline 9. & 88 & 85 & 80 & 74 & 69 & 69 & 62 & 62 & 62 \\
\hline 10. & 83 & 80 & 75 & 69 & 64 & 60 & 60 & 55 & 51 \\
\hline 11. & 88 & 70 & 52 & 30 & 25 & 46 & 28 & 15 & 8 \\
\hline 12. & 94 & 80 & 66 & 55 & 47 & 25 & 20 & 10 & 5 \\
\hline 13. & 87 & 82 & 75 & 60 & 55 & 44 & 40 & 34 & 30 \\
\hline 14. & 87 & 80 & 71 & 55 & 30 & 30 & 55 & 46 & 40 \\
\hline 15. & 87 & 75 & 69 & 60 & 56 & 40 & 36 & 29 & 25 \\
\hline 16. & 83 & 76 & 71 & 71 & 71 & 65 & 59 & 55 & 55 \\
\hline 17. & 87 & 80 & 72 & 66 & 59 & 47 & 42 & 35 & 35 \\
\hline Mean & 78.07 & 74.14 & 68.35 & 61.28 & 54.35 & 49.57 & 46.57 & 40.92 & 38 \\
\hline
\end{tabular}

Table 3: showing every month VAS scores ( $\mathrm{mm}$ ) of stiffness

\begin{tabular}{|c|c|c|c|c|c|c|c|c|c|}
\hline Case no. & Base line & $1^{\text {st }}$ & $2^{\text {nd }}$ & $3^{\text {rd }}$ & 4th & 5 th & 6th & 7th & 8th \\
\hline 1. & --- & --- & --- & --- & --- & --- & --- & --- & --- \\
\hline 2. & --- & --- & --- & --- & --- & --- & --- & --- & --- \\
\hline 3. & --- & --- & --- & --- & --- & --- & --- & --- & --- \\
\hline 4. & --- & --- & --- & --- & --- & --- & --- & --- & --- \\
\hline 5. & 40 & 40 & 35 & 31 & 31 & 28 & 24 & 20 & 20 \\
\hline 6. & --- & --- & --- & --- & --- & --- & --- & --- & --- \\
\hline 7. & --- & --- & --- & --- & --- & --- & --- & --- & --- \\
\hline 8. & --- & --- & --- & --- & --- & --- & --- & --- & --- \\
\hline 9. & 40 & 36 & 33 & 30 & 27 & 22 & 16 & 10 & 10 \\
\hline 10. & 70 & 64 & 60 & 55 & 55 & 51 & 46 & 40 & 30 \\
\hline
\end{tabular}




\begin{tabular}{|c|c|c|c|c|c|c|c|c|c|}
\hline 11. & 60 & 50 & 42 & 34 & 21 & 37 & 19 & 9 & 0 \\
\hline 12. & 90 & 81 & 73 & 65 & 50 & 32 & 25 & 16 & 10 \\
\hline 13. & 82 & 75 & 69 & 50 & 50 & 34 & 22 & 17 & 10 \\
\hline 14. & 78 & 72 & 60 & 45 & 33 & 55 & 38 & 32 & 25 \\
\hline 15. & 67 & 55 & 48 & 48 & 35 & 26 & 20 & 15 & 10 \\
\hline 16. & 62 & 50 & 44 & 44 & 40 & 36 & 30 & 24 & 20 \\
\hline 17. & 62 & 57 & 51 & 47 & 42 & 38 & 33 & 30 & 30 \\
\hline Mean & 65.1 & 58 & 51.5 & 44.9 & 38.4 & 35.9 & 27.3 & 21.3 & 16.5 \\
\hline
\end{tabular}

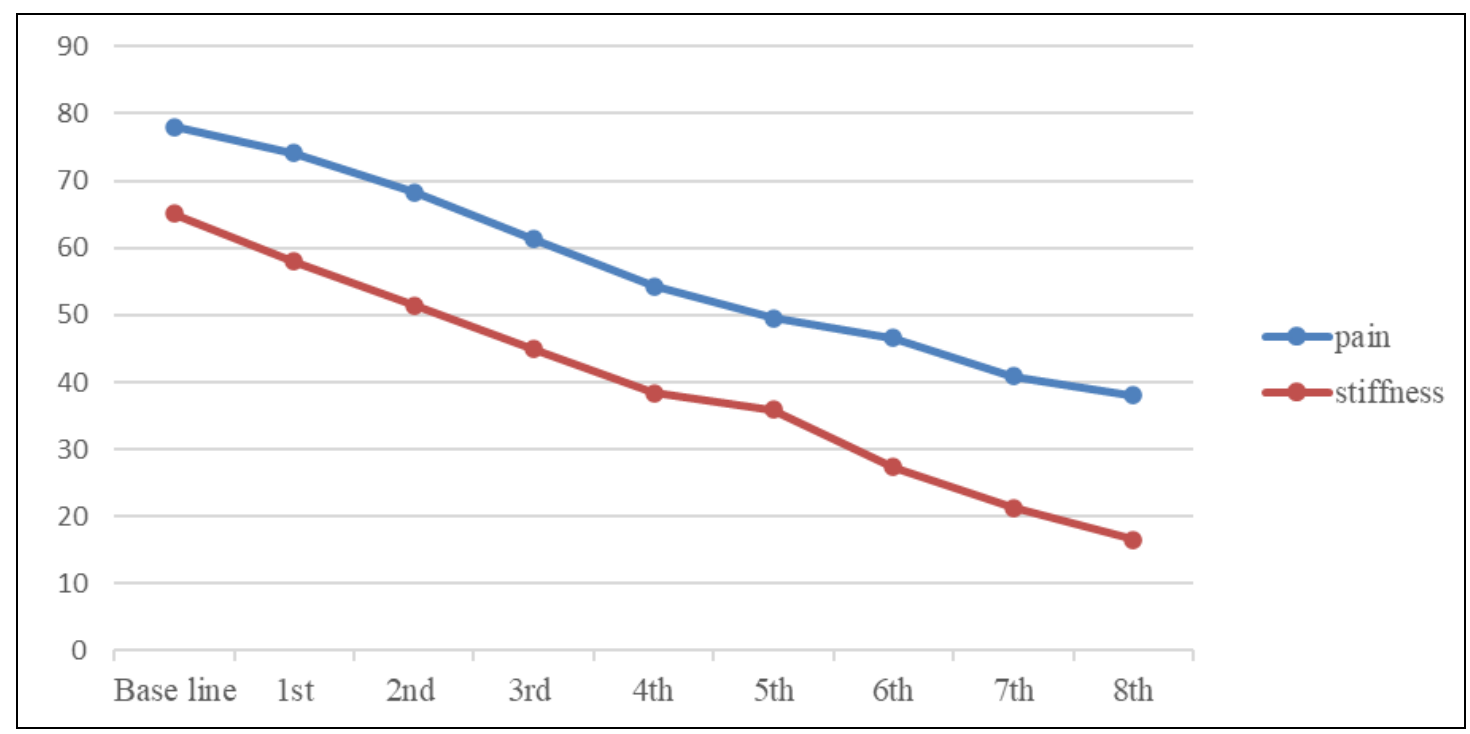

Fig 1: The course of pain and stiffness (mean values) of LS over 8 months

Table 4: Revised Oswestry low back pain disability questionnaire scores

\begin{tabular}{|c|c|c|c|c|c|c|}
\hline $\begin{array}{l}\text { Sl. } \\
\text { No. }\end{array}$ & $\begin{array}{c}\text { RODQ score } \\
\text { before } \\
\text { treatment } \\
\end{array}$ & $\begin{array}{c}\text { Mean RODQ score } \\
\text { before treatment }\end{array}$ & $\begin{array}{c}\text { RODQ score } \\
\text { after } \\
\text { treatment } \\
\end{array}$ & $\begin{array}{c}\text { Mean RODQ } \\
\text { score after } \\
\text { treatment }\end{array}$ & $\begin{array}{c}\text { Percentage }(\%) \text { of } \\
\text { disability before } \\
\text { treatment }\end{array}$ & $\begin{array}{c}\text { Percentage }(\%) \text { of } \\
\text { disability after } \\
\text { treatment }\end{array}$ \\
\hline 1 & 25 & \multirow{17}{*}{$\begin{array}{c}31.64 \\
\text { (excluding } 3 \\
\text { dropped out patients } \\
\text { i.e. } 4,6 \& 8 \text { ) }\end{array}$} & 8 & \multirow{17}{*}{13.57} & 50 & 16 \\
\hline 2 & 30 & & 35 & & 60 & 70 \\
\hline 3 & 34 & & 10 & & 68 & 20 \\
\hline 4 & 36 & & - & & 72 & - \\
\hline 5 & 28 & & 18 & & 56 & 36 \\
\hline 6 & 35 & & - & & 70 & - \\
\hline 7 & 32 & & 12 & & 64 & 24 \\
\hline 8 & 36 & & - & & 72 & - \\
\hline 9 & 34 & & 14 & & 68 & 28 \\
\hline 10 & 32 & & 10 & & 64 & 20 \\
\hline 11 & 35 & & 8 & & 70 & 16 \\
\hline 12 & 38 & & 8 & & 76 & 16 \\
\hline 13 & 30 & & 15 & & 60 & 30 \\
\hline 14 & 30 & & 14 & & 60 & 28 \\
\hline 15 & 32 & & 10 & & 64 & 20 \\
\hline 16 & 33 & & 18 & & 66 & 36 \\
\hline 17 & 30 & & 10 & & 60 & 20 \\
\hline
\end{tabular}

Table 5: Interpretation of disability scores

\begin{tabular}{|c|c|c|}
\hline \multirow{2}{*}{ Functional disability } & \multicolumn{2}{|c|}{ Number of patients } \\
\cline { 2 - 3 } & Before treatment & After treatment \\
\hline Minimal disability & 0 & 3 \\
\hline Moderate disability & 0 & 10 \\
\hline Severe disability & 2 & 0 \\
\hline Crippled & 15 & 1 \\
\hline
\end{tabular}




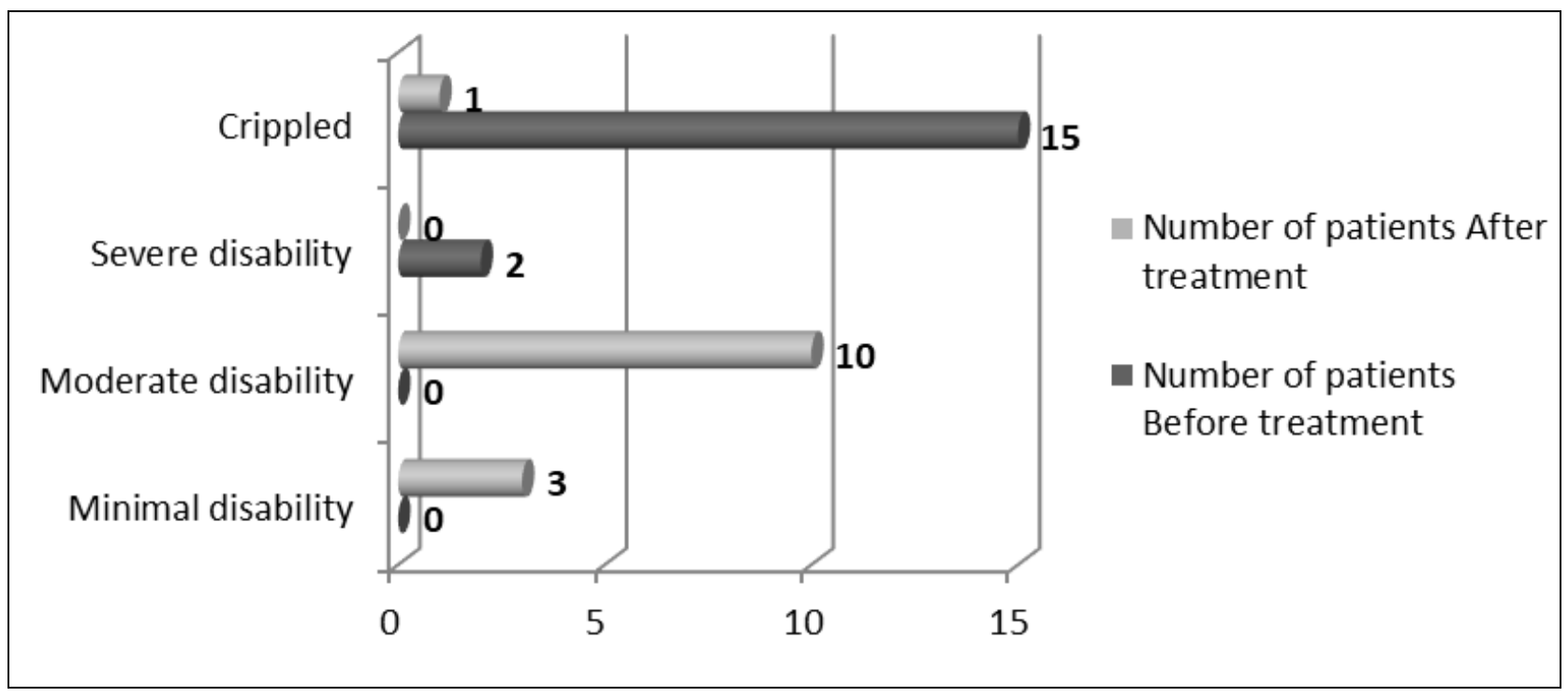

Fig 2: Interpretation of disability scores

Table 6: Statistical analysis of VAS score before and after treatment

\begin{tabular}{|c|c|c|c|c|c|c|c|c|}
\hline \multirow{2}{*}{$\begin{array}{l}\text { Sl. } \\
\text { No. }\end{array}$} & \multirow[b]{2}{*}{ Description } & \multirow{2}{*}{$\begin{array}{l}\text { Statistical } \\
\text { test }\end{array}$} & \multirow[b]{2}{*}{ 't' value } & \multirow[b]{2}{*}{ df } & \multicolumn{2}{|c|}{ Mean VAS score \pm SD } & \multirow{2}{*}{$\begin{array}{l}\text { Mean difference } \\
\text { with } 95 \% \mathrm{CI}\end{array}$} & \multirow{2}{*}{$\begin{array}{l}\text { Statistical } \\
\text { significant } \\
\text { at } p \leq 0.05\end{array}$} \\
\hline & & & & & At base line & $\begin{array}{c}\text { After } \\
\text { treatment }\end{array}$ & & \\
\hline 1 & $\begin{array}{l}\text { Comparison of VAS score } \\
\text { of pain before and after } \\
\text { treatment in } 14 \text { patients }\end{array}$ & $\begin{array}{l}\text { Paired ' } t \text { ' } \\
\text { test }\end{array}$ & 4.804384 & 13 & $\begin{array}{c}78.07 \pm \\
13.72\end{array}$ & $38 \pm 25.55$ & $40.07(22.05-58.09)$ & Yes \\
\hline 2 & $\begin{array}{l}\text { Comparison of VAS score } \\
\text { of stiffness before and after } \\
\text { treatment in } 10 \text { patients }\end{array}$ & $\begin{array}{l}\text { Paired 't' } \\
\text { test }\end{array}$ & 8.013409 & 9 & $\begin{array}{l}65.10 \pm \\
16.35\end{array}$ & $16.5 \pm 10.01$ & $48.60(34.88-62.32)$ & Yes \\
\hline 3 & $\begin{array}{l}\text { Comparison RODQ scores } \\
\text { before and after treatment }\end{array}$ & $\begin{array}{c}\text { Paired 't' } \\
\text { test }\end{array}$ & 8.033693 & 13 & $31.64 \pm 3.20$ & $13.57 \pm 7.06$ & $18.07(13.21-22.93)$ & Yes \\
\hline
\end{tabular}

\section{Discussion}

This case series of LS showed a significant improvement in the pain and stiffness of the back pain. Among the 17 cases $53 \%$ are female and $47 \%$ are male. Mean VAS score for pain reduced from $78.07 \pm 13.72 \mathrm{~mm}$ (baseline)to $38 \pm 25.55$ mm (end);95\% CI: 22.05 - 58.09; $P<0.05$. Stiffness reduced from $65.10 \pm 16.35$ ((baseline) to $16.50 \pm 10.01 \mathrm{~mm}$ (end); 95\% CI: $34.88-62.32 ; P<0.05$. Mean RODQ score reduced from $31.64 \pm 3.20$ (baseline) to $13.57 \pm 7.06$ (end); $95 \% \mathrm{CI}$ : $13.21-22.93 ; P<0.05$. Results of paired ' $t$ 'testshave been shown in table no. 6 .

The individualized homoeopathic medicines are effective in managing pain, stiffness and functional disability of patients with lumbar spondylotic changes. Therefore, the utilization of homoeopathic medicine in such cases may help the patients by avoiding use of pain killers regularly and thus saving them from adverse effects of the drugs. Documentation of improvement was done for all the fourteen cases having variations in respect of age, gender, duration of illness and diagnosis. Validated outcome measures were used for such documentation and the improvements were attributed to the homoeopathic medicines selected on the basis of individualization.

An acceptable external validity of the findings of this case series is expected as the study setting was similar to those obtained in routine clinical practice. Internal validity is expected to be low due to lack of a comparator group. Chances of information bias were negated by the prospective nature of the study and selection bias was minimized by the consecutive nature of recruitment of the cases.
The conclusions are limited however, because the study does not contain a control group for comparison of placebo effect. Absence of a comparator group prohibited any hypothesis from being tested and any direct causal inference cannot be made regarding the efficacy or effectiveness of the treatment method. However, this case series may be useful for the purpose of hypothesis generation which can be tested in future by a parallel group explanatory or pragmatic trial with optimum sample size.

\section{References}

1. Gupta K, Mamidi P. Ayurvedic management of lumbar spondylosis with spondylolisthesis: A case report. Journal of Pharmaceutical and Scientific Innovation [Internet]. 2014 Nov-Dec[cited 2019; 3(6):533-535. DOI: $10.7897 / 2277-4572.036211$

2. Damayanthie Fernando KP, Thakar AB, Shukla VD. Clinical efficacy of Eranda Muladi Yapana Basti in the management of Kati Graha (Lumbar spondylosis). An International Quarterly Journal of Research in Ayurveda [Internet]. 2013 Jan-Mar[cited 2019; 34(1):36-41. DOI: 10.4103/0974-8520.115444

3. Bjarnason I, Hayllar J, Macpherson AJ, Russell AS. Side effects of nonsteroidal anti-inflammatory drugs on the small and large intestine in humans. Gastroenterology [Internet]. 1993 Jun [cited 2019 Apr 26];104(6):1832-1847.

https://doi.org/10.1016/0016-5085(93)90667-2

4. Abram SE, C O'Connor T. Complications associated with epidural steroid injections. Regional Anesthesia\& Pain Medicine [Internet]. 1996 Mar [cited 2019; 
21(2):149-62.

http://dx.doi.org/10.1136/rapm-00115550-199621020-

00012

5. Wong WH, Litwic AE, Dennison EM. Complementary medicine use in rheumatology: A review. World J Rheumatol [Internet].2015 Nov[cited 2019; 5(3):142147.

DOI: 10.5499/wjr.v5.i3.142

6. Foltz V, Pierre YS, Rozenberg S, Rossiggnol M, Bourgeois P, Joseph L et al. Use of complementary and alternative therapies by patients with self-reported chronic back pain: a nationwide survey in Canada. Joint Bone Spine[Internet].2005 Dec[cited 2019; 72(6): 571577. https://doi.org/10.1016/j.jbspin.2005.03.018

7. Jadhav PM, Jadhav MP, Shelke P, Sharma Y, Nadkar M. Assessment of use of complementary alternative medicine and its impact on quality of life in the patients attending rheumatology clinic, in a tertiary care centre in India. Indian Journal of Medical Sciences[Internet]. 2011 Feb[cited 2019; 65(2):27-34.

DOI: $10.4103 / 0019-5359.103961$

8. Gmünder R, Kissling R. The effect of classical homeopathy compared to standard physiotherapy in the treatment of chronic low back pain. Z Orthop your Grenzgeb [internet]. 2002[cited 2019; 140(5):503-508. DOI: 10.1055 / s-2002-34004.

9. Witt CM, Lüdtke R, Baur R, Willich SN. Homeopathic treatment of patients with chronic low back pain: A prospective observational study with 2 years' followup.Clin J Pain [Internet]. 2009 May [cited 2019; 25(4):334-9.

doi: 10.1097/AJP.0b013e31819050bb.

10. Raj PP, Bayula B. Homeopathic Treatment of Lumbar Spondylosis: An Observational Study. American Journal of Homeopathic Medicine 2015; 108(3):104110.

11. Haefeli M, Elfering A. Pain assessment. Eur Spine J[Internet]. 2006[cited 2019; 15:17-24. DOI 10.1007/s00586-005-1044-x

12. https://www.ipmhealthcare.com/storage/app/media/Os westry.pdf

13. Page SJ, Shawaryn MA, Cernich AN, Linacre JM. Scaling of the revised Oswestry low back pain questionnaire. Archives of Physical Medicine and Rehabilitation[Internet].Nov 2002[cited 2019; 83(11):1579-1584. https://doi.org/10.1053/apmr.2002.34604

14. Aithala JP, Kumar S, Aithal S, Kotian SM. Development of a Modified Disability Questionnaire for Evaluating Disability Caused by Backache in India and Other Developing Countries. Asian Spine J 2018; 12(6):1106-1116. Doi: 10.31616/asj.2018.12.6.1106

15. Hart DL, Stratford PW, Werneke MW, Deutscher D, Wang YC. Lumbar Computerized Adaptive Test and Modified Oswestry Low Back Pain Disability Questionnaire: Relative Validity and Important Change. Journal of Orthopaedic \& Sports Physical Therapy [Internet]. 2012[cited 2019; 42(6):541-551. DOI:10.2519/jospt.2012.3942 